A mutation in the $\alpha$ tropomyosin gene TPM3 associated with autosomal dominant nemaline myopathy NEM1

Nigel Laing, Stephen Wilton, Patrick Akkari, Shellie Dorosz, Karyn Boundy, Chris Kneebone, Peter Blumbergs, Sue White, Hugh Watkins, Donald Love \& Eric Haan

Nature Genetics 9, 75-79 (1995)

The primer (5'-CTTGTCTAACTTCAGCATAGGC-3') stated to introduce an Nsil site into the normal TPM3 sequence is in fact a primer which introduces a StuI site into the mutate TPM3 sequence. This works very well for detecting the mutation since incomplete digestion does not give a control pattern. The correct primer for introducing a Nsil site into the normal TPM3 sequence is: 5'-CTTGTCTAACTTCAGCATATG-3'.

\section{Stability of an expanded trinucleotide repeat in the androgen receptor gene in transgenic mice}

Peter M. Bingham, Marion O. Scott, Suping Wang, Michael J. McPhaul, Elizabeth M. Wilson, James Y. Garbern, Diane E. Merry \& Kenneth H. Fischbeck Nature Genetics 9, 191-196 (1995)

On page 192 in the second paragraph under the cross heading "AR transgene expression", Figure $3 a$ should read Figure $3 b$, and Figure $3 b$ should read Figure $3 a$. (The figure legend correctly refers to $a$ and $b$. )

Also, the authors unintentionally omitted the following credit in the Acknowledgements section: Injection of transgene fragments and implantation of ova was carried out by the Transgenic Core Facility of the University of Pennsylvania's School of Medicine.

\section{Inherited microdeletions in the Angelman and Prader-Willi syndromes define an imprinting centre on human chromosome 15}

Karen Buiting, Shinji Saitoh, Stephanie Gross, Bärbel Bittrich, Stuart Schwartz, Robert D. Nicholls \& Bernhard Horsthemke

Nature Genetics 9, 395-400 (1995)

In the legend to Fig. 3, the last sentence should read: The patients lack the paternal band, whereas the father of family $U$ and the paternal grandmother of family $S$ lack the maternal band. 